\title{
A Novel Pre-Amplifier for WDM Systems Based on a Fiber Optical Parametric Amplifier
}

\author{
Yu Liang, Jia Li, P. C. Chui, and Kenneth K. Y. Wong* \\ Photonic Systems Research Laboratory, Department of Electrical and Electronic Engineering, \\ The University of Hong Kong, Hong Kong, kywong@eee.hku.hk.
}

\begin{abstract}
We demonstrate a novel scheme to improve receiver sensitivity of using a fiber optical parametric amplifier. The power penalty can be improved by $3.9 \mathrm{~dB}$ and the receiver sensitivity can achieve $-42 \mathrm{dBm}$ when $B E R=10^{-9}$.
\end{abstract}

\section{INTRODUCTION}

Fiber-based optical parametric amplifier (OPA) with femtosecond response time [1], high gain [2] and wide gain bandwidth [3] over several communication bands, has been demonstrated to be a candidate as a receiver pre-amplifier [4], based on the pump depletion effect in OPA. However, the intrinsic pump depletion effect confines its application to a single channel (i.e. signal plus pump). So the OPA-assisted pre-amplifier will be of great significance if it can be extended to WDM systems.

In this paper, we propose and demonstrate a novel preamplifier to improve receiver sensitivity by using dual-end detection of OPA for WDM systems.

\section{PRINCIPLE AND EXPERIMENTAL SETUP}

The principle of pre-amplifier based on OPA is shown in Fig. 1. By launching a weak signal together with a strong pump into nonlinear medium, both the amplification of weak signal and generation of new component called idler will occur by transferring power from the strong pump. As a result, the pattern on signal can be transcribed to idler, while the pump can be depleted simultaneously. By feeding both the amplified signal and idler into a photodetector (PD), constructive superposition can enhance the amplitude of the signal. Therefore, an output electrical signal with higher voltage swing is obtained. Implementing this scheme in each channel of WDM systems, extra amplification can be obtained. Note that previous work focused on combining the depleted pump and signal to enhance the signal swing [4].

The experimental setup is shown in Fig. 2, with part (a) as the pre-amplifier. The nonlinear medium used for OPA is a spool of 1-km highly-nonlinear dispersion-shifted fiber (HNLDSF) with nonlinear coefficient $\gamma \approx 14 \mathrm{~W}^{-1} \mathrm{~km}^{-1}$ and zerodispersion wavelength $\lambda_{0} \approx 1560 \mathrm{~nm}$. The OPA pump is supplied from a tunable laser source (TLS1) with emission wavelength at $1561.1 \mathrm{~nm}$. It is phase-modulated with $10-\mathrm{Gb} / \mathrm{s}$ $2^{31}-1$ pseudo-random binary sequence (PRBS) for stimulated Brillouin scattering (SBS) suppression. The phase-dithered pump is then amplified by two erbium-doped fiber amplifiers (EDFA1 and EDFA2) to $27.7 \mathrm{dBm}$ with a tunable band-pass filter (TBPF1) between them to reduce the amplified spontaneous emission (ASE) noise before entering into

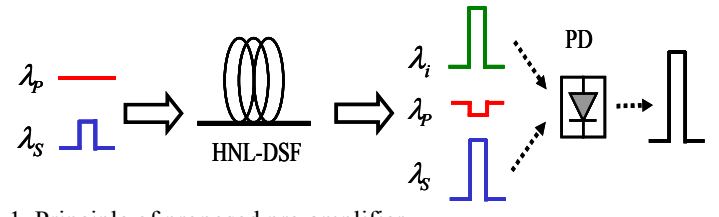

Fig. 1. Principle of proposed pre-amplifier.

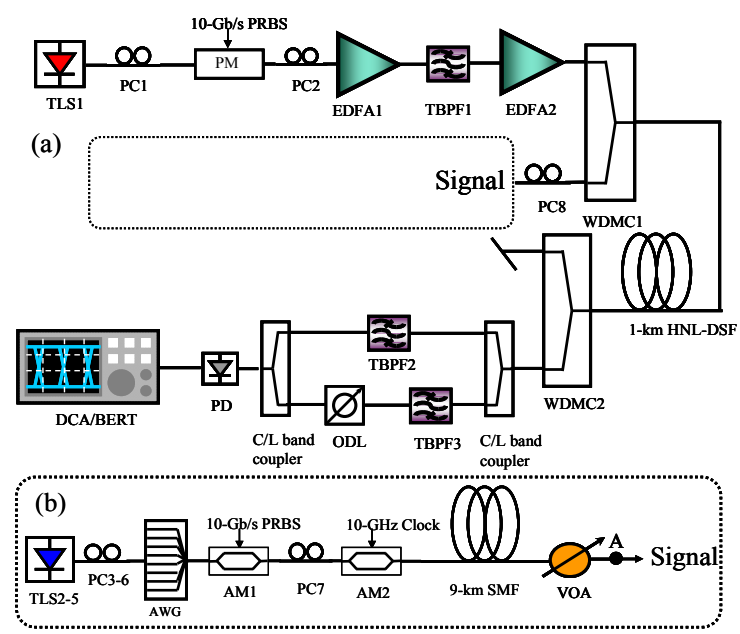

Fig. 2. Experimental setup of (a) pre-amplifier and (b) four WDM channels transmitter.

EDFA2. In Fig. 2(b), the dash line box shows the signal part. Four signal laser sources (TLS2-5) with wavelengths from $1545.3 \mathrm{~nm}$ to $1547.8 \mathrm{~nm}$ are combined by an arrayed waveguide grating (AWG) with channel spacing of $100 \mathrm{GHz}$. Then the four lightwaves are intensity-modulated by two Mach-Zehnder intensity modulators (MZ-IM) with 10-Gb/s $2^{31}-1$ PRBS and 10-GHz clock, respectively. A spool of 9$\mathrm{km}$ single mode fiber (SMF) is used to decorrelate the four channels. The pump and signals are combined using a WDM coupler (WDMC1) and launched into HNL-DSF for parametric amplification. Maximum OPA gain is attained by aligning the state of polarizations (SOPs) of signals and pump through polarization controllers PC2 and PC8. Then the pump is filtered out by WDMC2. The signals and idlers are split by a $\mathrm{C} / \mathrm{L}$ band coupler, while the corresponding signal and idler are filtered by TBPF2 and TBPF3. And the path length is compensated by optical delay lines (ODL). They are combined again using another $\mathrm{C} / \mathrm{L}$ band coupler. The output is monitored through digital communication analyzer (DCA) with a $30-\mathrm{GHz}$ bandwidth PD and bit-error rate tester (BERT) with $10-\mathrm{GHz}$ bandwidth PD. The sensitivity of the receiver is defined as the signal power measured at the input port of 

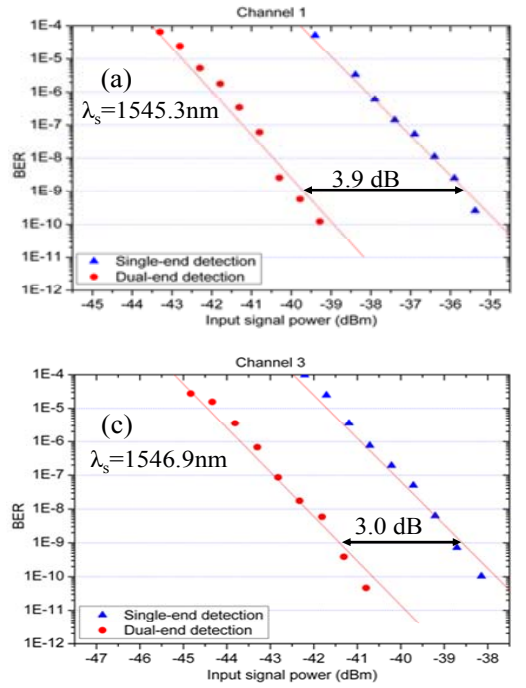

Fig.3. BER plots of (a) channe1, (b) channel 2, (c) channel 3, and (d) channel 4.

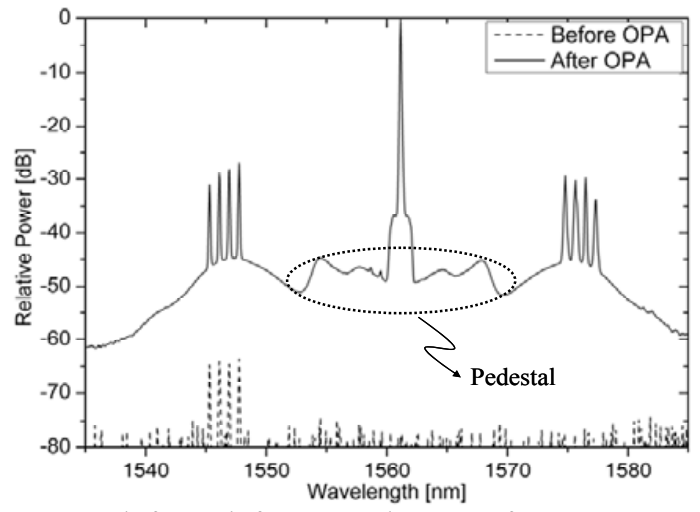

Fig. 4. Spectra before and after OPA at the output of HNL-DSF.

WDMC1 (denoted as point A in Fig. 2), which is controlled by a variable optical attenuator (VOA).

\section{RESUlts AND Discussion}

In order to quantify the benefit of dual-end detection, biterror rate (BER) of single- and dual-end detection schemes for four WDM channels are measured and shown in Fig. 3. From channel 1 to 4 at wavelength from $1545.3 \mathrm{~nm}$ to $1547.8 \mathrm{~nm}$ with spacing of $100 \mathrm{GHz}$, receiver sensitivities at $\mathrm{BER}=10^{-9}$ can reach $-35.6 \mathrm{dBm},-37.8 \mathrm{dBm},-38.5 \mathrm{dBm}$ and $-39 \mathrm{dBm}$ by single-end detection only using amplified signals. Their difference can be explained by the different parametric gain experienced by each channel as shown in Fig. 4, which shows the spectra at the output of the 1-km HNL-DSF. The dash line represents the four $10-\mathrm{Gb} / \mathrm{s}$ signals; while the solid line corresponds to the amplified signals. The parametric gains experienced by the four channels are $33 \mathrm{~dB}, 35 \mathrm{~dB}, 37 \mathrm{~dB}$ and $37 \mathrm{~dB}$, respectively, which can be measured from the spectra as shown in Fig. 4. The channel in high-gain region can achieve lower sensitivity. Note that the pedestal of pump is caused by the WDMC1. Using dual-end detection scheme, the receiver sensitivities can be improved by $3.9 \mathrm{~dB}, 3.3 \mathrm{~dB}, 3.0$ $\mathrm{dB}$, and $3.1 \mathrm{~dB}$, respectively. The improvement is contributed
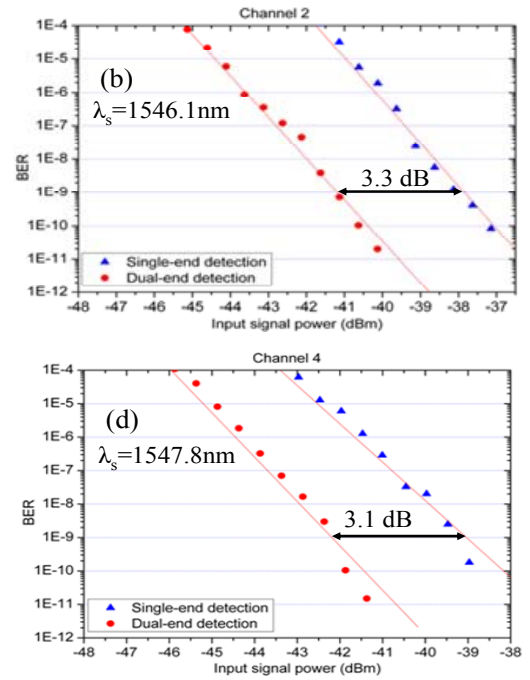

by the increase in signal swing from idlers. The output signals of pre-amplifier can obtain extra power from idlers while input signal power remains the same.

\section{CONCLUSION}

We demonstrated a novel receiver sensitivity improvement scheme for $10-\mathrm{Gb} / \mathrm{s}$ RZ-OOK signal using constructive superposition of signals and idlers in OPA for WDM systems by dual-end detection. Low receiver sensitivity down to -42 $\mathrm{dBm}$ was realized and power penalties were improved by more than $3 \mathrm{~dB}$ in all channels compared to the single-end counterpart through signal swing enhancement. This scheme would be useful for improving performance of OPA-assisted receiver in WDM systems.

\section{ACKNOWLEDGE}

This work was partially supported by grants from the Research Grants Council of the Hong Kong Special Administrative Region, China (Projects No. HKU 7172/07E and HKU 7179/08E). The authors would also like to acknowledge Sumitomo Electric Industries for providing the HNL-DSF.

\section{REFERENCES}

[1] M. E. Marhic, Fiber Optical Parametric Amplifiers, Oscillators and Related devices. Cambridge, UK: Cambridge Univ. Press, 2007.

[2] T. Torounidis, P. A. Andrekson and B. E. Olsson, "Fiber-optical parametric amplifier with 70-dB gain," IEEE Photon. Technol. Lett., vol. 18, no. 10, pp. 1194-1196, 2006.

[3] M. E. Marhic, K. K. Y. Wong and L. G. Kazovsky, "Wide-band tuning of the gain spectrum of one-pump fiber optical parametric amplifiers," IEEE J. Select. Topics Quantum Electron., vol. 10, no. 5. pp. 1133-1141, 2004.

[4] B. P. P. Kuo, K. Shimizu, and K. K. Y wong, "Receiver Sensitivity Improvement for ON-OFF Keying Signal by using Optical Parametric Amplifier and Balanced Detection,". in Proc. IEEE/LEOS WTM'08, paper MB3.3. 